\title{
Inhibition of PP2A by hesperetin may contribute to Akt and ERK1/2 activation status in cortical neurons
}

Article

Accepted Version

Creative Commons: Attribution-Noncommercial-No Derivative Works 4.0

Vauzour, D., Corsini, S., Müller, M. and Spencer, J. P. E. (2018) Inhibition of PP2A by hesperetin may contribute to Akt and ERK1/2 activation status in cortical neurons. Archives of Biochemistry and Biophysics, 650. pp. 14-21. ISSN 0003-9861 doi: https://doi.org/10.1016/j.abb.2018.04.020 Available at https://centaur.reading.ac.uk/80696/

It is advisable to refer to the publisher's version if you intend to cite from the work. See Guidance on citing.

To link to this article DOI: http://dx.doi.org/10.1016/j.abb.2018.04.020

Publisher: Elsevier

All outputs in CentAUR are protected by Intellectual Property Rights law, including copyright law. Copyright and IPR is retained by the creators or other copyright holders. Terms and conditions for use of this material are defined in the End User Agreement.

www.reading.ac.uk/centaur 
Central Archive at the University of Reading

Reading's research outputs online 
Inhibition of PP2A by hesperetin may contribute to Akt and ERK1/2 activation status in cortical neurons

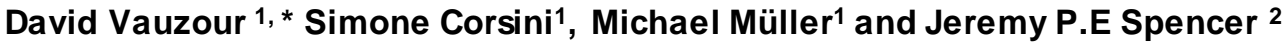

1 Norwich Medical School, University of East Anglia, Norwich NR4 7UQ, UK

2 Hugh Sinclair Unit for Human Nutrition, School of Chemistry, Food and Pharmacy, University of Reading, Reading RG2 6AP, UK

* To whom correspondence should be addressed: Dr David Vauzour, Norwich Medical School, University of East Anglia, Norwich NR4 7UQ, UK. D.Vauzour@uea.ac.uk ; Tel.: +441603 591732 


\section{ABSTRACT}

Flavonoids and their metabolites are well reported to modulate the activation/phosphorylation of various cellular kinases, such as ERK1/2 and JNK, although the mechanism by which they do so is unclear. In this study, we investigated the impact of flavanones on the activation of P/3K/Akt and ERK $1 / 2$ and determine whether this is mediated, in part, by the inhibition of phosphatases. Primary cortical neurons were exposed to physiological concentrations of hesperetin and the phosphorylation status of the kinases PI3K/Akt and ERK1/2 and the phosphatases PP2A and PTEN were assessed by immunoblotting after $30 \mathrm{~min}$. Exposure to $100-300 \mathrm{nM}$ hesperetin led to significant increases in the phosphorylation of ERK1/2 and Akt and significant decreases in PP2A levels and enzyme activity. Using in silico docking, hesperetin was found to fit into the active site of PP2A interacting within the hydrophobic cage of the catalytic unit. These data suggest a potential mechanism by which flavanones may lead to increased activation of ERK1/2 and Akt, commonly observed in cell models. Their interaction with and inhibition of cellular phosphatases counteract normal physiological regulation of signaling pathway activation thus facilitating and/or maintaining the activation status of ERK $1 / 2$ and Akt, important regulators of brain functions.

KEYWORDS: Flavonoids, brain, signaling pathways, phosphatase, MAP Kinase 


\section{BACKGROUND}

Accumulating evidence suggests that diet and lifestyle can play an important role in delaying the onset or halting the progression of age-related neurodegenerative diseases. A growing number of dietary intervention studies in humans and animals, in particular those using flavonoid-rich diets have been proposed to exert a multiplicity of neuroprotective actions within the brain, including a preservation of cognitive performance with ageing [1-3], a delay in the onset of Alzheimer's disease $[4,5]$ and a reduction in the risk of developing Parkinson's disease [6, 7]. Their neuroprotective potential has been shown in both oxidative stress- $[8,9]$ and $A \beta$-induced-induced neuronal death models [10]. Evidence also supports the beneficial and neuromodulatory effects of flavonoid-rich blueberry and grape extracts, particularly in connection with age-related cognitive decline and Alzheimer's disease [11, 12].

Flavonoids are well reported to cross the blood-brain-barrier (BBB) and have been detected in areas of the brain such as hippocampus, cerebral cortex, cerebellum and striatum [13-16], which are important for learning and memory formation and are also adversely affected by aging and neurodegenerative disorders [17, 18]. Hesperetin, a flavanone-type flavonoid, present as hesperidin (hesperetin 7-O- $\beta$-rutinoside) in the peel and pulp of a wide variety of citrus fruits and herbs [19], is known to appear in the circulation as predominantly hesperetin glucuronide and sulfate conjugates [20, 21], with studies reporting peak plasma concentrations ranging from 63 to $1500 \mathrm{nM}[22,23]$. Nanomolar concentrations of flavonoids have previously been reported in the brain in animal models following oral ingestion [14, 15, 24] and hesperetin itself has been shown to have high apparent permeability (Papp) across the in vitro BBB model, mainly due to its lipophilic nature [25] and has been suggested to accumulate in the brain at nanomolar concentrations [26, 27]. Independent of this, a flavanone glucuronides may to cross the BBB using specific transporters, as has been previously described for morphine 6-glucuronide [28]. Furthermore, hydrolysis of the glucuronide moiety may also occur following release of beta-glucuronidase (EC 3.2.1.31), an enzyme present in high concentrations in the endoplasmic reticulum and in the lysosomal fraction of most cells, an event that may be exacerbated by localized inflammation. Collectively, these data indicate that flavanones might be capable of transversing the BBB, localizing within the brain and exerting direct neuroprotective and neuromodulatory actions. 
The precise cellular mechanisms underlying the actions of flavonoids are still unknown but are thought to involve, in addition to their free radicals scavenging activity, the modulation of protein and lipid kinase signaling pathways [29, 30]. Previous investigations have highlighted that modulations of MAP kinase and PI3K/Akt signaling are central to mediating the cellular effects of flavonoids [29-31]. For example, we previously reported that hesperetin was effective at preventing neuronal apoptosis via a mechanism involving the activation/phosphorylation of both Akt/protein kinase $B$ and extracellular signal-regulated kinase 1 and 2 (ERK1/2) at physiological concentrations [32]. Furthermore, the flavanone naringenin was able to inhibit p38 mitogen-activated protein kinase (MAPK) phosphorylation and downstream signal transducer and activator of transcription-1 (STAT-1) in LPS/IFN-gamma stimulated primary mixed glial cells [33]. In addition to MAPK signaling, recent evidence is also suggestive that flavonoids are able to modulate reversible phosphorylation through protein phosphatase activity, an important mechanism of signal transduction in eukaryotic cells [34]. For example, the flavanol EGCG was previously reported to strongly inhibit protein phosphatase-1 (PP1) while showing a ten-fold lower activity toward the close homologue PP2A [35]. More recently, procyanidins were reported to negatively affect the activity of the PP1 and PRL phosphatases in regenerating livers [36]. These data not only indicate that flavonoids may exert cellular effects via direct interactions with MAPK and PI3K/Akt signaling proteins but may also be capable of cellular actions through protein phosphatases modulation. However, data related to the impact of physiologically relevant flavonoids on neuronal protein phosphatases remain to be investigated.

In this study we investigated the ability of the flavanone hesperetin to modulate neuronal signaling pathways at physiologically relevant concentrations, known in humans to be in the nanomolar to low micromolar range. We show that this dietary-derived compound has the capacity to activate both ERK and Akt signaling pathways, and that this kinase activation may be modulated through the inhibition of the protein phosphatase PP2A. In support of this, we show the molecular docking of hesperetin with the PP2A protein within the phosphatase active site of the catalytic unit. These results offer new avenues to the modulatory effect of flavonoids on neuronal signaling.

\section{MATERIALS AND METHODS}




\section{Reagents}

Reagents were from Sigma Chemicals Co. (Poole, Dorset, UK) unless otherwise stated. Hesperetin was obtained from Extrasynthese (Genay, France). Antibodies used were: anti-ACTIVE MAPK (ERK1/2), anti-pAkt (Ser-473), ERK1/2, Akt were from New England Biolabs (Hitchin, Hertfordshire, UK). PP2A and PTEN antibodies were from Insight Biotechnology Ltd (Wembley, UK). Horseradish peroxidase-conjugated goat anti-rabbit secondary antibody (Sigma, Poole, UK), ECL reagent and Hyperfilm-ECL were purchased from Amersham Biosciences (Amersham, UK). Ultrapure water (18.2 $M \Omega . c m$ ) passed through a purification system (Purite Ltd., Oxon, UK) was used for all purposes.

\section{Neuronal culture and treatments}

Primary cultures of mouse cortical neurons were prepared as described previously [32, 37]. Neurons were plated onto 6-well Nunc multi-well plates that had been pre-coated overnight with poly-Lornithine and then with $10 \%$ heat inactivated fetal bovine serum (Gibco-BRL, Paisley, UK) for $2 \mathrm{~h}$. Following removal of the final coating solution cells were plated $\left(10^{6} / \mathrm{ml}\right)$ in a serum-free medium composed of a mixture of DMEM and F-12 nutrient (1:1 v/v) supplemented with glucose $(33 \mathrm{mM})$, glutamine $(2 \mathrm{mM})$, sodium bicarbonate $(6.5 \mathrm{mM})$, HEPES $(\mathrm{pH} 7.4,5 \mathrm{mM})$, streptomycin $(100 \mu \mathrm{g} / \mathrm{ml})$ and penicillin (60 Ul/ml) (all Gibco-BRL). A mixture of hormones and salts composed of insulin (25 $\mu \mathrm{g} / \mathrm{ml})$, transferrin $(100 \mu \mathrm{g} / \mathrm{ml})$, putrescine $(60 \mu \mathrm{g} / \mathrm{ml})$, progesterone $(20 \mathrm{nM})$ and sodium selenate $(30$ nM) (all from Sigma) was also added to the culture medium. Cells were cultured at $37^{\circ} \mathrm{C}$ in a humidified atmosphere of $95 \%$ air and $5 \% \mathrm{CO}_{2}$ and after 5 to 7 days the vast majority of cells were neuronal (> 98\%) with $<2 \%$ astrocytes as determined by -tubulin and GFAP immunocytochemistry respectively [38]. To investigate the effect of the flavanone on signaling pathways, neurons were incubated for 30 minutes with hesperetin $(0-0.3 \mu \mathrm{M})$. This time point was selected based on our previous investigation [32].

\section{Phosphatase assay}

Phosphatase activity of mouse cortical neuronal cells was quantitated using the DuoSet $\AA$ IC Active PP2A assay kits (R\&D Systems, DYC3309) by measuring the dephosphorylation of a synthetic phospho-peptide, DLDVPIPGRFDRRVS(PO3)VAAE by active phosphatase PP2A. Briefly, cortical 
neurons plated in 6-cm Petri dishes were rinsed twice in TBS and lysed in the phosphatase buffer (50 mM HEPES, 0.5\% NP-40 (pH 7.5), 0.1 mM EGTA, 0.1 mM EDTA, $120 \mathrm{mM} \mathrm{NaCl}$ ) containing protease inhibitors $(25 \mu \mathrm{g} / \mathrm{mL}$ Leupeptin, $25 \mu \mathrm{g} / \mathrm{mL}$ Pepstatin, $2 \mu \mathrm{g} / \mathrm{mL}$ Aprotinin, $1 \mathrm{mM}$ PMSF). Proteins were extracted on ice with periodic vortexing for $15 \mathrm{~min}$. Cells were thoroughly solubilised by passing the lysates through a 19-gauge needle 20 times and cleared by centrifugation at $12,000 \times \mathrm{g}$ for $5 \mathrm{~min}$ at $4^{\circ} \mathrm{C}$. Diluted supernatants were then incubated with $200 \mu \mathrm{M}$ of synthetic phosphopeptide DLDVPIPGRFDRRVS(PO3)VAAE on a rocking platform at $30 \mathrm{rpm}$ for 30 minutes at $37^{\circ} \mathrm{C}$ in a buffer consisting of $50 \mathrm{mM}$ Tris (pH 7.5), $10 \mathrm{mM} \mathrm{MgCl} 2,0.02 \%$ Brij-35, $1 \mathrm{mg} / \mathrm{mL}$ BSA without or with $20 \mathrm{nM}$ okadaic acid. Following incubation, Malachite Green was added to the mixture and incubated for $20 \mathrm{~min}$ at room temperature to allow color development. Absorbance was measured at $620 \mathrm{~nm}$ using the Genios Tecan microplate reader. Phosphate released was determined by comparing absorbance to a standard phosphate curve. To calculate PP2A specific activity, total PP2A levels were also quantified using the DuoSet® IC Total PP2A assay kits (R\&D Systems, DYC1653).

\section{Immunoblotting}

Following exposures, neurons were washed with ice-cold PBS (+ EGTA $200 \mathrm{mM}$ ) and lysed on ice using Tris $(50 \mathrm{mM})$, Triton $\mathrm{X}-100(0.1 \%), \mathrm{NaCl}(150 \mathrm{mM})$ and EGTA/EDTA (2 mM), containing mammalian protease inhibitor cocktail (1:100 dilution), sodium pyrophosphate (1 mM), PMSF (10 $\mathrm{mg} / \mathrm{ml})$, sodium vanadate $(1 \mathrm{mM})$ and sodium fluoride $(50 \mathrm{mM})$. Lysed cells were scraped and left on ice to solubilise for $45 \mathrm{~min}$. Lysates were centrifuged at $1,000 \mathrm{xg}$ for $5 \mathrm{~min}$ at $4^{\circ} \mathrm{C}$ to remove unbroken cell debris and nuclei. Protein concentration in the supernatants was determined by the bicinchoninic acid (BCA) Protein Assay (Thermo-Fisher Scientific, Loughborough, UK). Samples were incubated for $5 \mathrm{~min}$ at $95^{\circ} \mathrm{C}$ in boiling buffer (final conc. $62.5 \mathrm{mM}$ Tris, pH 6.8, $2 \%$ SDS, 5\% 2-mercaptoethanol, $10 \%$ glycerol and $0.0025 \%$ bromophenol blue). Boiled samples (20-40 $\mu \mathrm{g} / \mathrm{lane}$ ) were run on $9-12 \%$ SDS-polyacrylamide gels and proteins were transferred to nitrocellulose membranes (Hybond-ECL; Amersham) by semi-dry electroblotting $(1.5 \mathrm{~mA} / \mathrm{cm} 2)$. The nitrocellulose membrane was then incubated in a blocking buffer (20mM Tris, pH 7.5, $150 \mathrm{mM} \mathrm{NaCl}$; TBS) containing 4\% (w/v) skimmed milk powder for $45 \mathrm{~min}$ at room temperature followed by $2 \times 5$ min washes in TBS supplemented with 0.05\% (v/v) Tween 20 (TTBS). Blots were then incubated with either anti-ACTIVE MAPK (ERK1/2) 
pAb (1:1000), anti-phospho-Akt (Ser 473) pAb (1:1000), anti-ERK1/2 (1:1000), anti Akt (1:1000), antiPTEN pAb (1:1000) or anti-PP2A pAb (1:1000) in TTBS containing 1\% (w/v) skimmed milk powder (antibody buffer) overnight at room temperature on a three dimensional rocking table. The blots were washed $2 \times 10 \mathrm{~min}$ in TTBS and incubated with goat anti-rabbit $\lg G$ conjugated to HRP (1:1000 dilution) for $60 \mathrm{~min}$. Finally, blots were washed $2 \times 10 \mathrm{~min}$ in TTBS rinsed in TBS and exposed to ECL-reagent for 1-2 min and developed. Bands were analyzed using Bio-Rad Quantity One 1-D Analysis software. Molecular weights of the bands were calculated from comparison with pre-stained molecular weight markers (MW 27,000 - 180,000 and MW 6,500 - 45,000, Sigma) that were run in parallel with the samples. The equal loading and efficient transfer of proteins was confirmed by staining the nitrocellulose with Ponceau Red (Sigma).

\section{In Silico Docking}

Docking calculations were conducted with SwissDock, a protein-small molecule docking web service [39]. SwissDock is based on the docking software EADock DSS, whose algorithm consists of the following steps: i) many binding modes are generated either in a box (local docking) or in the vicinity of all target cavities (blind docking); ii) simultaneously, their CHARMM energies are estimated on a grid; iii) the binding modes with the most favorable energies are evaluated with FACTS, and clustered; iv) the most favorable clusters can be visualized online and downloaded on a personal computer (http://swissdock.vital-it.ch/). UCSF Chimera (a visualization system for exploratory research and analysis) was used for molecular structure viewing [40]. The crystal structure was obtained from the PDB with the accession code 2NYM_C [41]. Hesperetin 3D structure was obtained by the ZINC 15 Database [42, 43] (code 39092).

\section{Statistical analysis}

All results are expressed as means \pm SEM of three separate experiments unless otherwise stated. The statistical evaluation of the results was performed by one-way analysis of variance (ANOVA) followed by a Bonferroni's multiple comparison test using GraphPad Prism version 5 (GraphPad Software, San Diego, CA, USA). Significance was defined as $p<0.05$. 


\section{RESULTS}

\subsection{Hesperetin stimulates Akt and ERK phosphorylation in cortical neurons}

To investigate the phosphorylation state of ERK1/2 and Akt/PKB, phospho-specific antibodies were employed that recognize the dually phosphorylated motif pTEpY within activated ERK1/2, and the phosphorylated Ser473 of Akt/PKB respectively. Exposure of cortical neurons to hesperetin $(0.3 \mu \mathrm{M}$; $30 \mathrm{~min}$ ) resulted in a significant increase in Akt phosphorylation at Ser473 $[F(2,3)=15.45 ; P=$ 0.0364], an event known to be essential for full activation of the kinase (Figure 1A). No significant changes were observed at smaller concentrations. We also determined the effects of hesperetin on the ERK1/2 pathway, by immunoblotting protein samples from neurons that had been exposed to increasing concentrations of the flavanone $(0-0.3 \mu \mathrm{M} ; 30 \mathrm{~min})$. Exposure of neurons to hesperetin resulted in a marked increase in ERK phosphorylation relative to basal levels, as demonstrated by a robust increase in the relative intensity of the immuno-detectable bands relating to phospho-ERK1 (44 $\mathrm{kDa}$ ) and phospho-ERK2 (42 kDa). Similar to Akt/PKB phosphorylation state, we observed the largest increase in ERK activation at $0.3 \mu \mathrm{M}[\mathrm{F}(2,6)=7.219 ; \mathrm{P}=0.0007]$, although ERK1/2 phosphorylation was also significantly increased in neurons exposed to $0.1 \mu \mathrm{M}[\mathrm{F}(2,6)=7.219 ; \mathrm{P}=0.0306]$ (Figure 1B). Parallel blots were run and probed with antibodies that detected total levels of ERK $1 / 2$ and total levels of Akt/PKB, demonstrating no modification in the total amount of proteins and an equal amount of loading (Figure 1A and 1B). Assessment of the other mitogen activated protein kinases (i.e. JNK and p38) did not reveal any modification in their phosphorylation state under these experimental conditions (data not shown).

\subsection{Hesperetin decreases PP2A activity in cortical neurons}

Since phosphatases can modulate the activities of several kinases in vitro and in vivo, such as ERK and Akt (reviewed in [44]), we investigated the impact of hesperetin on PP2A and PTEN, by immunoblotting protein samples from neurons that had been exposed to increasing concentrations of the flavanone $(0-0.3 \mu \mathrm{M} ; 30 \mathrm{~min})$. Exposure of cortical neurons to hesperetin $(0.3 \mu \mathrm{M} ; 30 \mathrm{~min})$ resulted in a significant decrease in PP2A levels relative to vehicle treated cells $[F(2,3)=9.210 ; P=$ 
0.0470] (Figure 2A). Although we observed a small increase in PTEN immunoreactivity following hesperetin incubation, this effect didn't reach significance $[F(2,6)=4.351 ; P=0.2471]$.

To further investigate the impact of hesperetin on PP2A, we quantified the phosphatase activity using both the Active and Total PP2A DuoSet IC activity assays. Incubating cortical neurons with hesperetin $(0-0.3 \mu \mathrm{M} ; 30 \mathrm{~min})$ led to a robust decrease in phosphate released (Figure $3 \mathrm{~A}$ ), indicating strong inhibition of PP2A activity $[F(2,9)=13.32 ; P=0.0020]$. To determine non-specific activity, cortical neurons were also incubated with Okadaic acid (OA; $20 \mathrm{nM})$, a known PP2A inhibitor. Following incubation with OA, PP2A activity was significantly decreased $[F(1,9)=67.40 ; P<0.0001]$ suggesting specific inhibition of the enzyme. These results are consistent with previous reports demonstrating specific inhibition of PP2A activity by OA [45]. Next, we determined PP2A specific activity by calculating the ratio of phosphate released per minute to total PP2A protein. Although there was a loss of total PP2A protein with hesperetin treatment (data not shown), a significant decrease in the specific activity of the enzyme was observed $[F(2,6)=20.17 ; P=0.0022]$ (Figure 3B). These data correlate well with the relative total amounts of PP2A detected by western blot, showing a significant decrease in PP2A immunoreactive bands.

\subsection{Molecular docking of Hesperetin on PP2A}

The PP2A core enzyme consists of a $36 \mathrm{kDa}$ catalytic subunit, or $\mathrm{C}$ subunit, and a $65 \mathrm{kDa}$ scaffolding protein, known as the A or PR65 subunit [46]. Previous investigations looking into OA binding to PP2A revealed a binding pocket located in the active site of PP2A catalytic unit. On one end of the binding pocket, four amino acids in the catalytic subunit of PP2A, Gln122, Ile123, His191, and Trp200, form a hydrophobic cage, which accommodates the hydrophobic end of OA. On the other end of the binding pocket, Leu243, Tyr265, Cys266, Arg268, and Cys269 make multiple van der Waals interactions with the hydrophobic portion of the bound toxin [47].

In order to gain insight into the putative binding mode of hesperetin with PP2A catalytic subunit, it was docked with a crystallographic structure of human PP2A using SwissDock. The crystallographic structure was obtained from the Protein Data Bank (PDB), accession code 2NYM_C [41]. The docking model predicted by SwissDock indicates that hesperetin is able to fit into the active site of 
PP2A (Fig 4A and B). In particular, we observed interaction within the hydrophobic cage at two main sites (site A and B) representing the overall binding pocket of the catalytic unit. In particular, hesperetin interacts with residues of site A (Pro190) and B (Leu243 and Hsd252; Fig 4C) where it creates hydrogen bonds. In addition, hesperetin also established hydrophobic links with the ligand rings through interaction with the site B surface residues Val244, Asn249 and Trp250 (Fig 4D). Predicted binding energies for hesperetin-PP2A complex are summarized in Table 1.

\section{DISCUSSION}

Flavonoids have been proposed to exert beneficial effects in a multitude of diseases, including cancer, cardiovascular disease and neurodegenerative disorders [48-50]. Increasingly they have been observed to exert their cellular effects via the modulation of cell signaling pathways, such as the mitogen activated protein kinase (MAPK) and the PI3K pathways, two essential signaling cascades that regulate multiple processes in the central nervous system including proliferation, survival, metabolism and cell migration [51]. For example, flavanols have been demonstrated to inhibit stress activated extracellular signal-regulated kinase 1 and 2 (ERK1/2) and c-Jun N terminal kinase (JNK) signaling pathways resulting in the protection of neurons from apoptosis [52]. Our current data indicate that exposure of primary cortical neurons to low nanomolar concentrations of hesperetin resulted in a significant increase in the phosphorylation of ERK1/2 and Akt at Ser473. These data are in agreement with our previous findings demonstrating that hesperetin was effective at modulating both Akt/protein kinase B and extracellular signal-regulated kinase 1 and 2 (ERK1/2) in neurons [24]. Although the uptake and distribution of dietary flavonoids within the brain is well documented, at least in animal studies, the concentration within specific neuronal tissues remains uncertain. Discrepancies mainly stem from the fact that studies reporting flavonoid brain uptake and concentrations often disregard residual blood as a potential confounder. However, data deriving from studies using neuronal tissue extracted from perfused animals suggests that flavonoids may localise in the brain at levels up to $1 \mathrm{nmol} / \mathrm{g}$ tissue [53] and in a non regio-specificic manner [54, 55]. Hesperetin itself has been shown to have high apparent permeability (Papp) across the in vitro BBB model, mainly due to its lipophilic nature [25] and was observed in the brain tissue at nanomolar concentrations [26, 27].

The concentrations used in our study reflect those observed in the brain in vivo. This is very 
important, as flavonoids have been shown to act as pro-apoptotic stimuli at higher concentrations. Indeed, we have previously shown that quercetin and its metabolites 3'-O-methyl quercetin and 4'-Omethyl quercetin inhibit protein kinase B/Akt (Akt) and ERK1/2 phosphorylation, an action underlying their pro-apoptotic action towards cortical neurons [56].

Reversible phosphorylation is a very important mechanism of signal transduction and is mediated by a series of kinases and phosphatases [34]. Amongst phosphatases, protein phosphatase 2A (PP2A) is one of the most conserved proteins and together with PP1, is responsible for up to $90 \%$ of all serine/threonine activity in a cell [57]. As a phosphatase, PP2A functions in many of the major cell signaling pathways including those that regulate the cell cycle, cell metabolism, cell migration and cell survival [58]. Numerous observations in vitro suggest that PP2A plays a major role in the downregulation of the ERK and Akt/PKB pathways, and that PP2A may be active at several levels of the signaling cascade [44]. Our results show that concomitant to ERK and Akt activation/phosphorylation, PP2A was significantly decreased by hesperetin treatment.

The balanced and highly dynamic interplay between kinases and phosphatases is critical for the control of intracellular signalling and consequently aberrant regulation of either protein kinases and/or phosphatases may play a causative role in diseases such as diabetes and neurological disorders [59]. As such both kinases and phosphatases may act as therapeutic in the aetiology of many diseases [60-65]. With respect to phosphatases, their regulation and/or activation status may lead to a variety of outcomes. For example, protein tyrosine phosphatase (PTP) 1B has been reported to negatively regulate insulin and leptin signalling making it a prime target for enhancing insulin sensitivity and controlling body mass [62]. In support of this, PTP1B-deficient mice show an enhanced insulin sensitivity, improved glycemic control, and resistance to high fat diet induced obesity [66, 67]. As such, if flavonoids such as hesperetin are capable of inhibiting PTP1B they may counteract insulin resistance via a mechanism that facilitates insulin-induced autophosphorylation of its receptor, thereby sustaining downstream kinase cascades that induce synthesis of the short-term energy storage glycogen and synthesis of fatty acids and proteins. Clearly the success of such inhibitors will depend on the effective absorption and selectivity of the flavonoid or its metabolites towards the 
phosphatase, however as indicated flavanones such as hesperetin are amongst the most brain bioavailable flavonoids and thus their activity in animal models is justified.

Many human clinical trials have indicated that flavonoid intervention, including that of citrus flavanones can induce reductions in blood pressure in hypertensive patients and increased blood flow in normotensive individuals [68]. These changes have been speculated to involve eNOS activation and nitric oxide synthesis. On the one hand, inhibitors of PP2A have been reported to increase eNOS activity via the reduced potential to dephosphorylate/deactivate serines 116,1176 and 1179 of the enzyme (Greif DM, Kou R, Michel T. Biochemistry. 2002 Dec 31;41(52):15845-53). Alternatively, the inhibition of PP2A will also limit the potential for dephosphorylation/deactivation of Akt at Thr-308 and Ser-473, thus promoting eNOS activity, nitric oxide bioavailability and optimum blood flow/pressure. Additionally, Thus flavanones may also lead to sustained activation of eNOS and nitric oxide generation in the vascular endothelium, which may underpin observations of increased flow-mediated dilatation and reductions in blood pressure in human clinical trials.

In addition to their role in modulating key intracellular signalling pathways involved in various aspects of physiology, phosphatases are thought to play a key role in neuronal function, including synaptic plasticity, apoptotic cell death [69] and neuroinflammation [70]. PP2A is speculated to be a potential biological target for the drug treatment of neurodegenerative diseases such as Parkinson's disease and Alzheimer's disease [69, 71]. Here, they may contribute to the abnormal phosphorylation of proteins, such as tau in the neurofibrillary tangles of Alzheimer's disease [72] or a-synuclein in the Lewy bodies of PD [73]. The abnormal hyperphosphorylation of such proteins is generally believed to lead to their mis-folding and aggregation [74]. Thus, effective inhibition of phosphatases such as PP2A may attenuate the progression of these neurodegenerative diseases. However, it remains unclear whether activation or inhibition of such phosphatases would be most therapeutic and also whether their inhibition may promote carcinogenesis. While it seems counterintuitive to inhibit a tumour suppressor such as PP2A, inhibitors such as LB-100 are known to sensitize tumours to chemotherapy and radiotherapy by blocking the removal of phosphate groups from proteins essential for cell cycle progression. Thus, when used with radio- or chemotherapy treatments, malignant cells will progress through the cell cycle without having their damaged DNA repaired thus shunting them to undergo tumour cell apoptosis. With respect to the ability of flavanones to inhibit other phosphatases, 
it is known that the function of protein-tyrosine phosphatases depends on a critical cysteine residue at the catalytic site, which forms a thiol-phosphate intermediate during catalysis $[75,76]$. Interaction with this thiol residue may therefore disrupt enzyme active. Whilst it is currently unknown whether flavanones may interact within this essential cysteine, under cellular conditions some flavonoids are capable of autoxidising and covalently binding to cellular thiols [77].

In summary, our findings suggest that observations relating to increases in kinases following exposure to flavonoids, including flavanones such as hesperetin may result in part due to the inhibition of phosphatases such as PP2A. Although the precise mechanism is yet to be established, and importantly the activity of phase II conjugated metabolites, such as glucuronides and sulphates, inhibition of phosphatases by flavanone aglycones may represent one mechanism by which flavonoids influence physiological function at the cellular level.

ACKNOWLEDGMENTS: This research was supported the Medical Research Council (grant ref. G0400278/N102) and is greatly appreciated.

AUTHOR CONTRIBUTIONS: DV and JPES conceived and designed the experiments. DV performed the neuronal culture, phosphatase assays and the immunoblotting. SC and MM contributed to the molecular docking. DV and JPES wrote the paper. All authors gave final approval of the version to be published. All authors read and approved the final manuscript.

CONFLICTS OF INTEREST: The authors have no conflict of interest to declare. 
TABLE

Table 1. Docking results of Hesperetin onto PP2A catalytic unit.

\begin{tabular}{cccc}
\hline Site & Ligand & Full Fitness & $\begin{array}{c}\text { Binding } \\
\text { energy, } \mathbf{\Delta G} \\
\text { (kcal/mol) }\end{array}$ \\
\hline A & Hesperetin & -1568.00 & -6.808 \\
B & Hesperetin & -1562.99 & -6.539 \\
\hline
\end{tabular}




\section{FIGURES CAPTION}

Figure 1: Hesperetin stimulates Akt and ERK1/2 phosphorylation in a concentration-dependent manner. Cortical neurons were exposed to increasing concentrations of hesperetin $(0.1 \mu \mathrm{M}-0.3 \mu \mathrm{M})$ for $30 \mathrm{~min}$ and the levels of phosphorylated Akt (Ser473), the dually phosphorylated ERK1/2 (pTEpY), total Akt and total ERK1/2 were measured by immunoblotting. (A) Representative immunoblots from a single experiment showing phosphorylated pAkt (Ser473) and total Akt; (B) phosphorylated ERK1/2 and total ERK1/2. Band intensities were determined by densitometric analysis using BioRad Quantity One 1-D Analysis. ${ }^{* *} p<0.001 ;{ }^{*} p<0.05$ : indicate significant increases in phosphorylation of Akt or ERK $1 / 2$ relative to vehicle treated cells, $n=3$

Figure 2: Hesperetin decreases PP2A levels in a concentration-dependent manner. Cortical neurons were exposed to increasing concentrations of hesperetin $(0.1 \mu \mathrm{M}-0.3 \mu \mathrm{M})$ for $30 \mathrm{~min}$ and the levels of PP2A, PTEN and beta actin were measured by immunoblotting. Representative immunoblots from a single experiment showing PP2A, PTEN and beta actin. Band intensities were determined by densitometric analysis using BioRad Quantity One 1-D Analysis and normalized against beta actin levels. ${ }^{*} p<0.05$ : indicate significant decrease in PP2A levels relative to vehicle treated cells, $n=3$.

Figure 3: Hesperetin decreases PP2A activity in cortical neurons. Cortical neurons were exposed to increasing concentrations of hesperetin $(0.1 \mu \mathrm{M}-0.3 \mu \mathrm{M})$ for 30 min and PP2A phosphatase activity of was quantitated using the DuoSet ${ }^{\circ}$ IC Active and Total PP2A assay kits. (A) Phosphate released in cortical neurons lysates following 30-minutes incubation with increased concentration of hesperetin (-OA). To determine non-specific activity, $20 \mathrm{nM}$ Okadaic acid (+ OA) was added to some of the samples and incubated for 15 minutes at room temperature. (B) Specific PP2A activity was calculated as the ratio of phosphate released to the amount of Total PP2A protein. ${ }^{\star \star \star} p<0.001 ;{ }^{* \star} p<0.01 ;{ }^{*} p$ $<0.05$ : indicate significant decreases in phosphatase activity relative to vehicle treated cells, $n=3$.

Figure 4: Molecular docking of hesperetin on PP2A. Panel (A), Hesperetin docked into the site A and B of PP2A catalytic subunit. Panels (B) and (C), the binding mode and the hydrogen bond network established by Hesperetin and neighboring residues in sites $A$ and $B$ (black lines in panel B): 
Hesperetin is hydrogen-bonded with the side chains of Leu243 and Hsd252 (green lines in panel (C)). Panel (D), interactions of Hesperetin rings with Val244, Asn249 and Trp250. Images are depicted using the Chimera software. 


\section{REFERENCES}

[1] L. Letenneur, C. Proust-Lima, G.A. Le, J.F. Dartigues, P. Barberger-Gateau, Flavonoid intake and cognitive decline over a 10-year period, Am J Epidemiol 165(12) (2007) 1364-1371.

[2] M.C. Morris, D.A. Evans, C.C. Tangney, J.L. Bienias, R.S. Wilson, Associations of vegetable and fruit consumption with age-related cognitive change, Neurology 67(8) (2006) 1370-6.

[3] S. Neshatdoust, C. Saunders, S.M. Castle, D. Vauzour, C. Williams, L. Butler, J.A. Lovegrove, J.P. Spencer, High-flavonoid intake induces cognitive improvements linked to changes in serum brainderived neurotrophic factor: Two randomised, controlled trials, Nutr Healthy Aging 4(1) (2016) 81-93.

[4] Q. Dai, A.R. Borenstein, Y. Wu, J.C. Jackson, E.B. Larson, Fruit and vegetable juices and Alzheimer's disease: the Kame Project, Am J Med 119(9) (2006) 751-9.

[5] G.M. Pasinetti, J. Wang, L. Ho, W. Zhao, L. Dubner, Roles of resveratrol and other grape-derived polyphenols in Alzheimer's disease prevention and treatment, Biochimica et biophysica acta 1852(6) (2015) $1202-8$.

[6] H. Checkoway, K. Powers, T. Smith-Weller, G.M. Franklin, W.T. Longstreth, Jr., P.D. Swanson, Parkinson's disease risks associated with cigarette smoking, alcohol consumption, and caffeine intake, Am J Epidemiol 155(8) (2002) 732-8.

[7] M. Chen, T. Wang, F. Yue, X. Li, P. Wang, Y. Li, P. Chan, S. Yu, Tea polyphenols alleviate motor impairments, dopaminergic neuronal injury, and cerebral alpha-synuclein aggregation in MPTPintoxicated parkinsonian monkeys, Neuroscience 286 (2015) 383-92.

[8] A. Gonzalez-Sarrias, M.A. Nunez-Sanchez, F.A. Tomas-Barberan, J.C. Espin, Neuroprotective Effects of Bioavailable Polyphenol-Derived Metabolites against Oxidative Stress-Induced Cytotoxicity in Human Neuroblastoma SH-SY5Y Cells, J Agric Food Chem 65(4) (2017) 752-758.

[9] M. Wang, Y.J. Li, Y. Ding, H.N. Zhang, T. Sun, K. Zhang, L. Yang, Y.Y. Guo, S.B. Liu, M.G. Zhao, Y.M. Wu, Silibinin Prevents Autophagic Cell Death upon Oxidative Stress in Cortical Neurons and Cerebral Ischemia-Reperfusion Injury, Mol Neurobiol 53(2) (2016) 932-943. 
[10] A. Telerman, R. Ofir, Y. Kashman, A. Elmann, 3,5,4 '-trihydroxy-6,7,3 '-trimethoxyflavone protects against beta amyloid-induced neurotoxicity through antioxidative activity and interference with cell signaling, Bmc Complem Altern M 17 (2017).

[11] A. Dal-Pan, S. Dudonne, P. Bourassa, M. Bourdoulous, C. Tremblay, Y. Desjardins, F. Calon, N. Consortium, Cognitive-Enhancing Effects of a Polyphenols-Rich Extract from Fruits without Changes in Neuropathology in an Animal Model of Alzheimer's Disease, J Alzheimers Dis 55(1) (2017) 115135.

[12] C. Rendeiro, D. Vauzour, R.J. Kean, L.T. Butler, M. Rattray, J.P.E. Spencer, C.M. Williams, Blueberry supplementation induces spatial memory improvements and region-specific regulation of hippocampal BDNF mRNA expression in young rats, Psychopharmacology 223(3) (2012) 319-330.

[13] K.P. Datla, M. Christidou, W.W. Widmer, H.K. Rooprai, D.T. Dexter, Tissue distribution and neuroprotective effects of citrus flavonoid tangeretin in a rat model of Parkinson's disease, Neuroreport 12(17) (2001) 3871-5.

[14] P.E. Milbury, W. Kalt, Xenobiotic metabolism and berry flavonoid transport across the blood-brain barrier, J Agric Food Chem 58(7) (2010) 3950-6.

[15] S. Passamonti, U. Vrhovsek, A. Vanzo, F. Mattivi, Fast access of some grape pigments to the brain, J Agr Food Chem 53(18) (2005) 7029-7034.

[16] K.A. Youdim, B. Shukitt-Hale, J.A. Joseph, Flavonoids and the brain: interactions at the bloodbrain barrier and their physiological effects on the central nervous system, Free Radic Biol Med 37(11) (2004) 1683-93.

[17] D. Vauzour, M. Camprubi-Robles, S. Miquel-Kergoat, C. Andres-Lacueva, D. Banati, P. Barberger-Gateau, G.L. Bowman, L. Caberlotto, R. Clarke, E. Hogervorst, A.J. Kiliaan, U. Lucca, C. Manach, A.M. Minihane, E.S. Mitchell, R. Perneczky, H. Perry, A.M. Roussel, J. Schuermans, J. Sijben, J.P. Spencer, S. Thuret, O. van de Rest, M. Vandewoude, K. Wesnes, R.J. Williams, R.S. Williams, M. Ramirez, Nutrition for the ageing brain: Towards evidence for an optimal diet, Ageing Res Rev 35 (2017) 222-240. 
[18] A.M. Fjell, L. McEvoy, D. Holland, A.M. Dale, K.B. Walhovd, I. Alzheimer's Disease

Neuroimaging, What is normal in normal aging? Effects of aging, amyloid and Alzheimer's disease on the cerebral cortex and the hippocampus, Prog Neurobiol 117 (2014) 20-40.

[19] J.A. Rothwell, J. Perez-Jimenez, V. Neveu, A. Medina-Remon, N. M'Hiri, P. Garcia-Lobato, C. Manach, C. Knox, R. Eisner, D.S. Wishart, A. Scalbert, Phenol-Explorer 3.0: a major update of the Phenol-Explorer database to incorporate data on the effects of food processing on polyphenol content, Database (Oxford) 2013 (2013) bat070.

[20] W. Mullen, M.A. Archeveque, C.A. Edwards, H. Matsumoto, A. Crozier, Bioavailability and metabolism of orange juice flavanones in humans: impact of a full-fat yogurt, J Agric Food Chem 56(23) (2008) 11157-64.

[21] M. Tomas-Navarro, F. Vallejo, E. Sentandreu, J.L. Navarro, F.A. Tomas-Barberan, Volunteer stratification is more relevant than technological treatment in orange juice flavanone bioavailability, $\mathrm{J}$ Agric Food Chem 62(1) (2014) 24-7.

[22] J.Q. Silveira, T.B. Cesar, J.A. Manthey, E.A. Baldwin, J. Bai, S. Raithore, Pharmacokinetics of flavanone glycosides after ingestion of single doses of fresh-squeezed orange juice versus commercially processed orange juice in healthy humans, J Agric Food Chem 62(52) (2014) 12576-84.

[23] F. Vallejo, M. Larrosa, E. Escudero, M.P. Zafrilla, B. Cerda, J. Boza, M.T. Garcia-Conesa, J.C. Espin, F.A. Tomas-Barberan, Concentration and solubility of flavanones in orange beverages affect their bioavailability in humans, J Agric Food Chem 58(10) (2010) 6516-24.

[24] M.M. Abd El Mohsen, G. Kuhnle, A.R. Rechner, H. Schroeter, S. Rose, P. Jenner, C. A. RiceEvans, Uptake and metabolism of epicatechin and its access to the brain after oral ingestion, Free Radic Biol Med 33(12) (2002) 1693-702.

[25] K.A. Youdim, M.S. Dobbie, G. Kuhnle, A.R. Proteggente, N.J. Abbott, C. Rice-Evans, Interaction between flavonoids and the blood-brain barrier: in vitro studies, J Neurochem 85(1) (2003) 180-92. 
[26] S. Mogami, C. Sadakane, M. Nahata, Y. Mizuhara, C. Yamada, T. Hattori, H. Takeda, CRF receptor 1 antagonism and brain distribution of active components contribute to the ameliorative effect of rikkunshito on stress-induced anorexia, Sci Rep-Uk 6 (2016).

[27] T.H. Tsai, Y.F. Chen, Determination of unbound hesperetin in rat blood and brain by microdialysis coupled to microbore liquid chromatography, J Food Drug Anal 8(4) (2000) 331-336.

[28] T.A. Aasmundstad, J. Morland, R.E. Paulsen, Distribution of morphine 6-glucuronide and morphine across the blood-brain barrier in awake, freely moving rats investigated by in vivo microdialysis sampling, J Pharmacol Exp Ther 275(1) (1995) 435-41.

[29] D. Vauzour, Dietary polyphenols as modulators of brain functions: biological actions and molecular mechanisms underpinning their beneficial effects, Oxid Med Cell Longev 2012 (2012) 914273.

[30] R.J. Williams, J.P. Spencer, C. Rice-Evans, Flavonoids: antioxidants or signalling molecules?, Free Radic Biol Med 36(7) (2004) 838-49.

[31] P.K. Bahia, M. Rattray, R.J. Williams, Dietary flavonoid (-)epicatechin stimulates phosphatidylinositol 3-kinase-dependent anti-oxidant response element activity and up-regulates glutathione in cortical astrocytes, J Neurochem 106(5) (2008) 2194-204.

[32] D. Vauzour, K. Vafeiadou, C. Rice-Evans, R.J. Williams, J.P. Spencer, Activation of pro-survival Akt and ERK1/2 signalling pathways underlie the anti-apoptotic effects of flavanones in cortical neurons, J Neurochem 103(4) (2007) 1355-67.

[33] K. Vafeiadou, D. Vauzour, H.Y. Lee, A. Rodriguez-Mateos, R.J. Williams, J.P. Spencer, The citrus flavanone naringenin inhibits inflammatory signalling in glial cells and protects against neuroinflammatory injury, Arch Biochem Biophys 484(1) (2009) 100-9.

[34] T. Hunter, Protein kinases and phosphatases: the yin and yang of protein phosphorylation and signaling, Cell 80(2) (1995) 225-36.

[35] A. Kiss, B. Becsi, B. Kolozsvari, I. Komaromi, K.E. Kover, F. Erdodi, Epigallocatechin-3-gallate and penta-O-galloyl-beta-D-glucose inhibit protein phosphatase-1, FEBS J 280(2) (2013) 612-26. 
[36] S. Stadlbauer, P. Rios, K. Ohmori, K. Suzuki, M. Kohn, Procyanidins Negatively Affect the Activity of the Phosphatases of Regenerating Liver, PloS one 10(7) (2015) e0134336.

[37] D. Vauzour, J.T. Pinto, A.J. Cooper, J.P. Spencer, The neurotoxicity of 5-S-cysteinyldopamine is mediated by the early activation of ERK $1 / 2$ followed by the subsequent activation of ASK $1 / \mathrm{JNK} 1 / 2$ pro-apoptotic signalling, The Biochemical journal 463(1) (2014) 41-52.

[38] M.S. Perkinton, T.S. Sihra, R.J. Williams, $\mathrm{Ca}(2+)$-permeable AMPA receptors induce phosphorylation of CAMP response element-binding protein through a phosphatidylinositol 3-kinasedependent stimulation of the mitogen-activated protein kinase signaling cascade in neurons, The Journal of neuroscience : the official journal of the Society for Neuroscience 19(14) (1999) 5861-74.

[39] A. Grosdidier, V. Zoete, O. Michielin, SwissDock, a protein-small molecule docking web service based on EADock DSS, Nucleic acids research 39(Web Server issue) (2011) W270-7.

[40] E.F. Pettersen, T.D. Goddard, C.C. Huang, G.S. Couch, D.M. Greenblatt, E.C. Meng, T.E. Ferrin, UCSF Chimera--a visualization system for exploratory research and analysis, Journal of computational chemistry 25(13) (2004) 1605-12.

[41] Y. Xu, Y. Xing, Y. Chen, Y. Chao, Z. Lin, E. Fan, J.W. Yu, S. Strack, P.D. Jeffrey, Y. Shi, Structure of the protein phosphatase 2A holoenzyme, Cell 127(6) (2006) 1239-51.

[42] J.J. Irwin, B.K. Shoichet, ZINC--a free database of commercially available compounds for virtual screening, Journal of chemical information and modeling 45(1) (2005) 177-82.

[43] T. Sterling, J.J. Irwin, ZINC 15--Ligand Discovery for Everyone, Journal of chemical information and modeling 55(11) (2015) 2324-37.

[44] T.A. Millward, S. Zolnierowicz, B.A. Hemmings, Regulation of protein kinase cascades by protein phosphatase 2A, Trends in biochemical sciences 24(5) (1999) 186-91.

[45] A.R. Fathi, A. Krautheim, S. Lucke, K. Becker, H. Juergen Steinfelder, Nonradioactive technique to measure protein phosphatase $2 \mathrm{~A}$-like activity and its inhibition by drugs in cell extracts, Analytical biochemistry 310(2) (2002) 208-14. 
[46] D.D. Green, S.I. Yang, M.C. Mumby, Molecular cloning and sequence analysis of the catalytic subunit of bovine type 2A protein phosphatase, Proceedings of the National Academy of Sciences of the United States of America 84(14) (1987) 4880-4.

[47] Y. Xing, Y. Xu, Y. Chen, P.D. Jeffrey, Y. Chao, Z. Lin, Z. Li, S. Strack, J.B. Stock, Y. Shi, Structure of protein phosphatase 2A core enzyme bound to tumor-inducing toxins, Cell 127(2) (2006) $341-53$.

[48] D. Del Rio, A. Rodriguez-Mateos, J.P. Spencer, M. Tognolini, G. Borges, A. Crozier, Dietary (poly)phenolics in human health: structures, bioavailability, and evidence of protective effects against chronic diseases, Antioxid Redox Signal 18(14) (2013) 1818-92.

[49] A. Rodriguez-Mateos, D. Vauzour, C.G. Krueger, D. Shanmuganayagam, J. Reed, L. Calani, P. Mena, D. Del Rio, A. Crozier, Bioavailability, bioactivity and impact on health of dietary flavonoids and related compounds: an update, Arch Toxicol 88(10) (2014) 1803-53.

[50] D. Vauzour, A. Rodriguez-Mateos, G. Corona, M.J. Oruna-Concha, J.P. Spencer, Polyphenols and human health: prevention of disease and mechanisms of action, Nutrients 2(11) (2010) 1106-31.

[51] M.C. Mendoza, E.E. Er, J. Blenis, The Ras-ERK and PI3K-mTOR pathways: cross-talk and compensation, Trends in biochemical sciences 36(6) (2011) 320-8.

[52] H. Schroeter, J.P. Spencer, C. Rice-Evans, R.J. Williams, Flavonoids protect neurons from oxidized low-density-lipoprotein-induced apoptosis involving c-Jun N-terminal kinase (JNK), c-Jun and caspase-3, The Biochemical journal 358(Pt 3) (2001) 547-57.

[53] S. Schaffer, B. Halliwell, Do polyphenols enter the brain and does it matter? Some theoretical and practical considerations, Genes Nutr (2011).

[54] C.M. Williams, M.A. El Mohsen, D. Vauzour, C. Rendeiro, L.T. Butler, J.A. Ellis, M. Whiteman, J.P. Spencer, Blueberry-induced changes in spatial working memory correlate with changes in hippocampal CREB phosphorylation and brain-derived neurotrophic factor (BDNF) levels, Free radical biology \& medicine 45(3) (2008) 295-305. 
[55] E.M. Janle, M.A. Lila, M. Grannan, L. Wood, A. Higgins, G.G. Yousef, R.B. Rogers, H. Kim, G.S. Jackson, L. Ho, C.M. Weaver, Pharmacokinetics and tissue distribution of 14C-labeled grape polyphenols in the periphery and the central nervous system following oral administration, J Med Food 13(4) (2010) 926-33.

[56] J.P. Spencer, C. Rice-Evans, R.J. Williams, Modulation of pro-survival Akt/protein kinase B and ERK $1 / 2$ signaling cascades by quercetin and its in vivo metabolites underlie their action on neuronal viability, J Biol Chem 278(37) (2003) 34783-93.

[57] J. Westermarck, W.C. Hahn, Multiple pathways regulated by the tumor suppressor PP2A in transformation, Trends Mol Med 14(4) (2008) 152-60.

[58] V. Janssens, J. Goris, Protein phosphatase 2A: a highly regulated family of serine/threonine phosphatases implicated in cell growth and signalling, The Biochemical journal 353(Pt 3) (2001) 41739.

[59] V.V. Vintonyak, A.P. Antonchick, D. Rauh, H. Waldmann, The therapeutic potential of phosphatase inhibitors, Curr Opin Chem Biol 13(3) (2009) 272-83.

[60] D. Barford, A.K. Das, M.P. Egloff, The structure and mechanism of protein phosphatases: insights into catalysis and regulation, Annu Rev Biophys Biomol Struct 27 (1998) 133-64.

[61] L. Bialy, H. Waldmann, Inhibitors of protein tyrosine phosphatases: next-generation drugs?, Angew Chem Int Ed Engl 44(25) (2005) 3814-39.

[62] B. Kasibhatla, J. Wos, K.G. Peters, Targeting protein tyrosine phosphatase to enhance insulin action for the potential treatment of diabetes, Curr Opin Investig Drugs 8(10) (2007) 805-13.

[63] N.K. Tonks, Protein tyrosine phosphatases: from genes, to function, to disease, Nat Rev Mol Cell Biol 7(11) (2006) 833-46.

[64] T. Yi, D. Lindner, The role and target potential of protein tyrosine phosphatases in cancer, Curr Oncol Rep 10(2) (2008) 114-21. 
[65] Z.Y. Zhang, Protein tyrosine phosphatases: prospects for therapeutics, Curr Opin Chem Biol 5(4) (2001) 416-23.

[66] M. Elchebly, P. Payette, E. Michaliszyn, W. Cromlish, S. Collins, A.L. Loy, D. Normandin, A. Cheng, J. Himms-Hagen, C.C. Chan, C. Ramachandran, M.J. Gresser, M.L. Tremblay, B.P. Kennedy, Increased insulin sensitivity and obesity resistance in mice lacking the protein tyrosine phosphatase 1B gene, Science 283(5407) (1999) 1544-8.

[67] L.D. Klaman, O. Boss, O.D. Peroni, J.K. Kim, J.L. Martino, J.M. Zabolotny, N. Moghal, M. Lubkin, Y.B. Kim, A.H. Sharpe, A. Stricker-Krongrad, G.I. Shulman, B.G. Neel, B.B. Kahn, Increased energy expenditure, decreased adiposity, and tissue-specific insulin sensitivity in protein-tyrosine phosphatase 1B-deficient mice, Mol Cell Biol 20(15) (2000) 5479-89.

[68] L. Testai, V. Calderone, Nutraceutical Value of Citrus Flavanones and Their Implications in Cardiovascular Disease, Nutrients 9(5) (2017).

[69] S.P. Braithwaite, M. Voronkov, J.B. Stock, M.M. Mouradian, Targeting phosphatases as the next generation of disease modifying therapeutics for Parkinson's disease, Neurochem Int 61(6) (2012) 899-906.

[70] E.C. Hirsch, S. Hunot, Neuroinflammation in Parkinson's disease: a target for neuroprotection?, Lancet Neurol 8(4) (2009) 382-97.

[71] J.M. Sontag, E. Sontag, Protein phosphatase 2A dysfunction in Alzheimer's disease, Front Mol Neurosci 7 (2014) 16.

[72] A.D. Alonso, T. Zaidi, M. Novak, H.S. Barra, I. Grundke-lqbal, K. lqbal, Interaction of tau isoforms with Alzheimer's disease abnormally hyperphosphorylated tau and in vitro phosphorylation into the disease-like protein, J Biol Chem 276(41) (2001) 37967-73.

[73] K. Arima, S. Hirai, N. Sunohara, K. Aoto, Y. Izumiyama, K. Ueda, K. Ikeda, M. Kawai, Cellular colocalization of phosphorylated tau- and NACP/alpha-synuclein-epitopes in lewy bodies in sporadic Parkinson's disease and in dementia with Lewy bodies, Brain Res 843(1-2) (1999) 53-61. 
[74] C.A. Ross, M.A. Poirier, Protein aggregation and neurodegenerative disease, Nat Med 10 Suppl (2004) S10-7.

[75] K.L. Guan, J.E. Dixon, Evidence for protein-tyrosine-phosphatase catalysis proceeding via a cysteine-phosphate intermediate, J Biol Chem 266(26) (1991) 17026-30.

[76] H. Cho, R. Krishnaraj, E. Kitas, W. Bannwarth, C.T. Walsh, K.S. Anderson, Isolation and structural elucidation of a novel phosphocysteine intermediate in the LAR protein tyrosine phosphatase enzymic pathway, Journal of the American Chemical Society 114(18) (1992) 7296-7298.

[77] J.P. Spencer, G.G. Kuhnle, R.J. Williams, C. Rice-Evans, Intracellular metabolism and bioactivity of quercetin and its in vivo metabolites, The Biochemical journal 372(Pt 1) (2003) 173-81. 
Figure 1

A
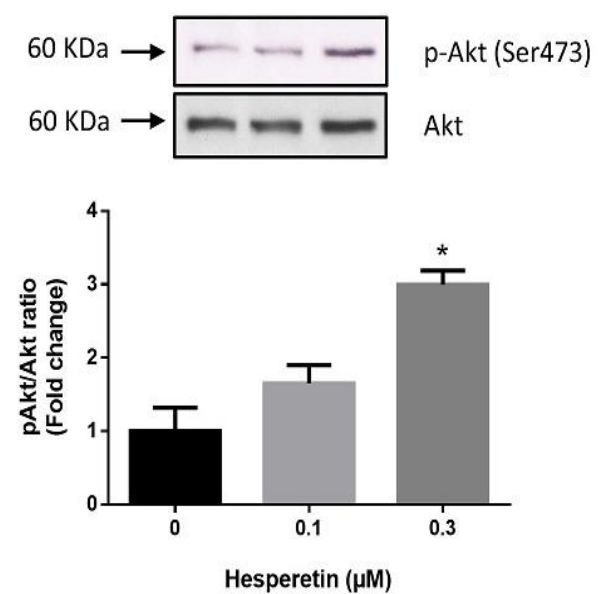

B
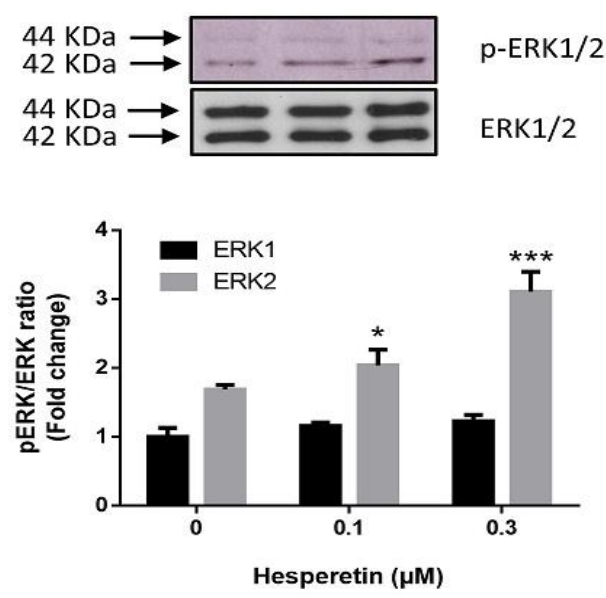
Figure 2
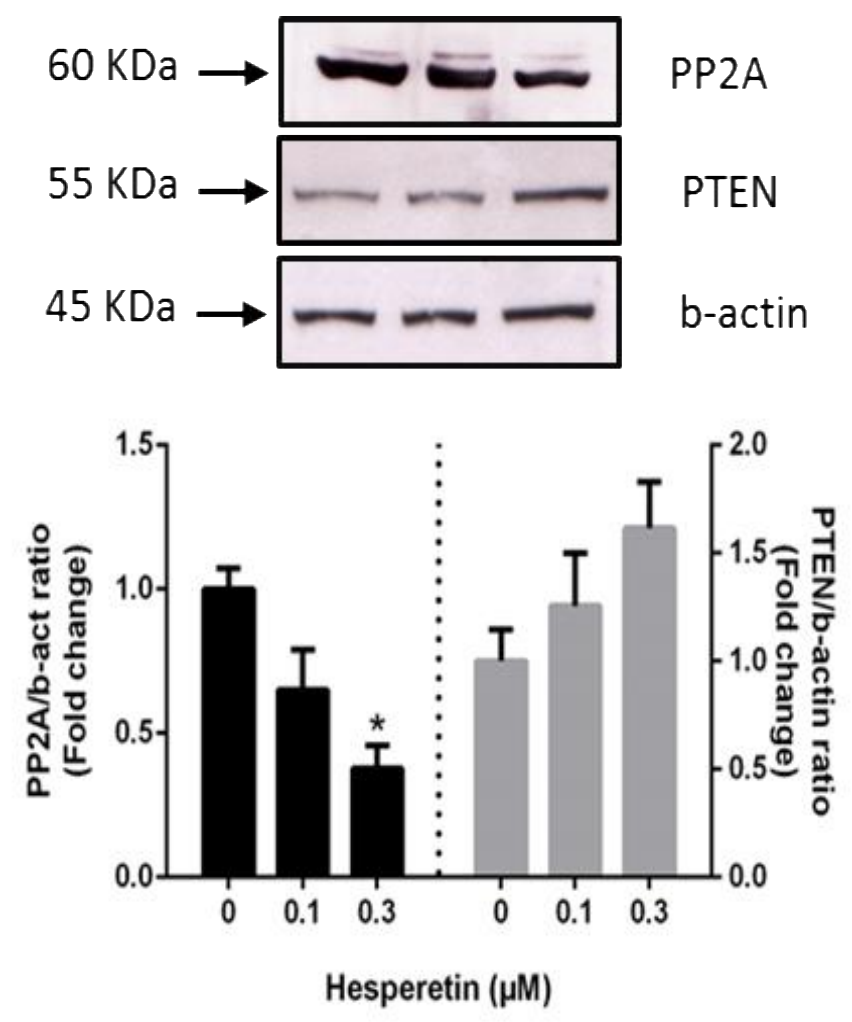


\section{Figure 3}

A

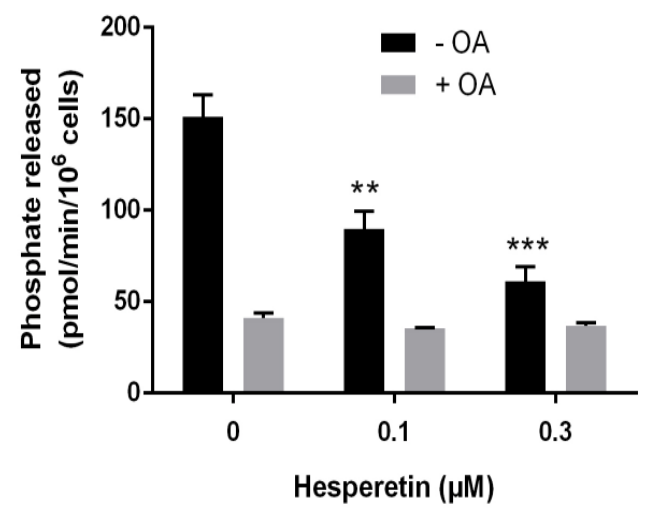

B

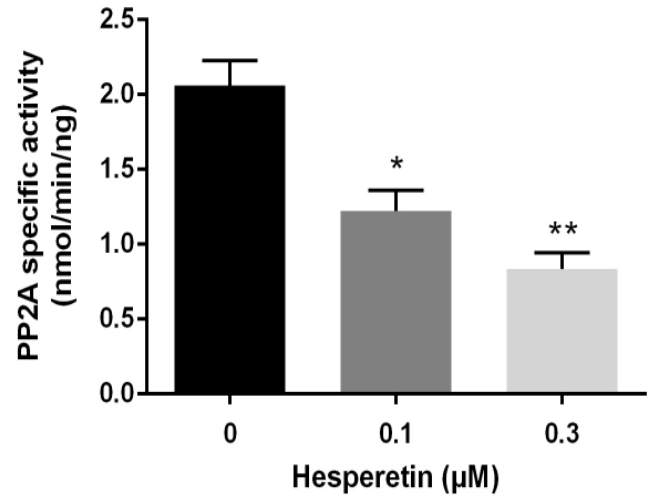


Figure 4
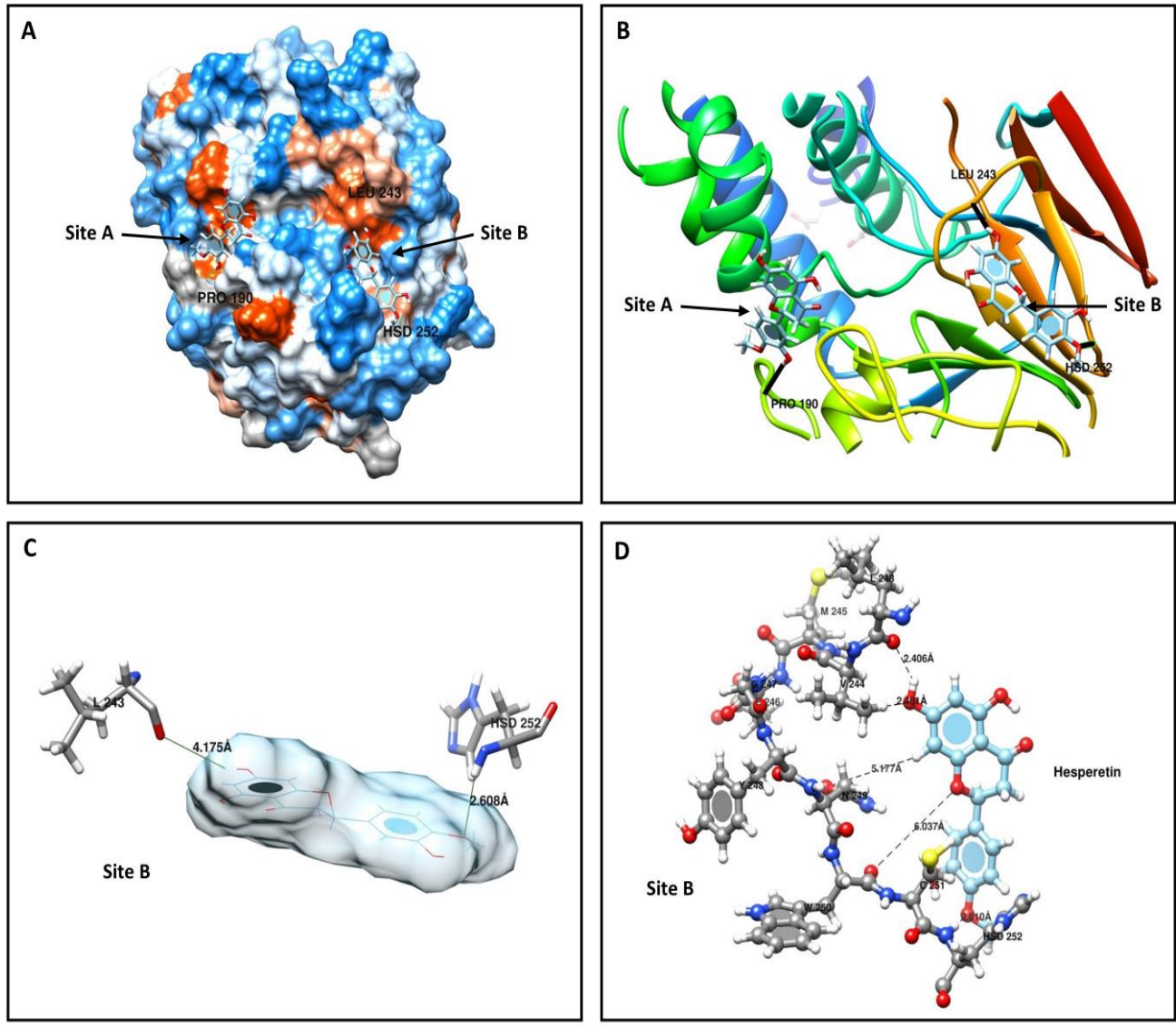